\title{
Spin Glass and ferromagnetism in disordered Cerium compounds
}

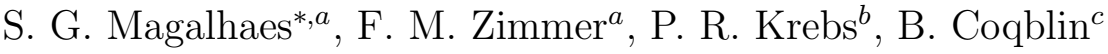 \\ ${ }^{a}$ Lab. de Mecânica Estatística e Teoria da Matéria Condensada, \\ Dep. Física, UFSM, 97105-900 Santa Maria, RS, Brazil \\ ${ }^{b}$ Instituto de Física e Matemática, Universidade Federal de Pelotas, \\ Caixa Postal 354, 96010-900 Pelotas, RS, Brazil \\ ${ }^{c}$ Laboratoire de Physique des Solides, Université Paris-Sud, \\ bâtiment 510, 91405 Orsay, France
}

\begin{abstract}
The competition between spin glass, ferromagnetism and Kondo effect is analysed here in a Kondo lattice model with an inter-site random coupling $J_{i j}$ between the localized magnetic moments given by a generalization of the Mattis model [15] which represents an interpolation between ferromagnetism and a highly disordered spin glass. Functional integral techniques with Grassmann fields have been used to obtain the partition function. The static approximation and the replica symmetric ansatz have also been used. The solution of the problem is presented as a phase diagram giving $T / J$ versus $J_{K} / J$ where $T$ is the temperature, $J_{K}$ and $J$ are the strengths of the intrasite Kondo and the intersite random couplings, respectively. If $J_{K} / J$ is small, when temperature is decreased, there is a second order transition from a paramagnetic to a spin glass phase. For lower $T / J$, a first order transition appears between the spin glass phase and a region where there are Mattis states which are thermodynamically equivalent to the ferromagnetism. For very low $T / J$, the Mattis states become stable. On the other hand, it is found as solution a Kondo state for large $J_{K} / J$ values. These results can improve the theoretical description of the well known experimental phase diagram of $C e N i_{1-x} C u_{x}$ $8,9,10,11$.
\end{abstract}

\section{Introduction}

The properties of many cerium or uranium compounds are well described by the Kondo-lattice model, with strong competition between the Kondo effect on each site and the Ruderman-Kittel-Yosida-Kasuya (RKKY) interaction between magnetic atoms at different sites. The role of disorder has been studied in disordered alloys containing cerium or uranium and different theories have been proposed. In the Kondo disordered model (KDM) [1, 2], disorder produces a

*ggarcia@ccne.ufsm.br 
broad distribution of Kondo temperatures and can be responsible for the deviation from the Fermi Liquid behavior found in some heavy fermion systems. Another theoretical approach is the Magnetic Griffths phase [3], where fluctuations of the magnetic clusters can produce Griffiths-McCoy singularities close to a Quantum Critical Point (QCP). On the other hand, we have studied within a mean field approximation the phase diagrams observed in disordered heavy fermion systems showing Kondo, spin glass and magnetically ordered phases [4, 5. 6] and we will discuss these models later on. Earlier studies have also suggested that a spin glass transition near a QCP could lead to a Non Fermi Liquid (NFL) behavior [7]. Our paper is an attempt to improve the theoretical description of the spin glass-Kondo-ferromagnetic competition in order to obtain a better agreement with the experimental situation of disordered cerium or uranium heavy fermion systems.

Spin glass and Kondo state have been observed together in several Cerium alloys like $C e N i_{1-x} C u_{x}$ [8, 9], $\mathrm{Ce}_{2} A u_{1-x} \mathrm{Co}_{x} S i_{3}$ [12] and in some disordered Uranium alloys such as $U C u_{5-x} P d_{x}$ [13] or $U_{1-x} L a_{x} P d_{2} A l_{3}$ [14]. The first case has been studied by bulk methods (see Refs. 8,9 ) and more recently by $\mu \mathrm{SR}$ spectroscopy [10] which gives local information about the spins configurations. The bulk probes have shown a presence of antiferromagnetic phase for low $\mathrm{Ni}$ content (for instance $x=0.9$ ). In the region $x \lesssim 0.2$, the Kondo effect becomes important producing magnetic moment reduction. For $0.8 \gtrsim x \gtrsim 0.4$, the same bulk probes have shown a presence of the spin glass like state intermediate in temperature between a ferromagnetic order (at lower temperature) and paramagnetism (at higher temperature). However, the $\mu \mathrm{SR}$ spectroscopy has shown in the region $0.8 \gtrsim x \gtrsim 0.4$ a scenario favoring the presence of a inhomogeneous "cluster spin glass" (or called equivalently "cluster glass" in Ref. 10]) rather than a standard spin glass. Quite recent measurements on the specific heat [11] has confirmed the emergence of a spin glass-like state and a percolative evolution to a ferromagnetic order at low temperatures.

There has been a theoretical attempt [5] to build up a global phase diagram based on a Kondo lattice model with a random Gaussian inter-site coupling among the localized spins with mean $2 J_{0} / N$ and standard deviation $\sqrt{8 \tilde{J}^{2} / N}$ ( $N$ is the number of sites). The spins operators have been given as bilinear combination of creation and destruction fermionic operators. The partition function has been found using path integral formalism within the static approximation and replica symmetry ansatz 4]. The results have shown that ferromagnetism, spin glass and a mixed phase (a solution with non-zero magnetization below the Almeida-Thouless line) have been obtained for small $J_{K} / \tilde{J}$ values and $J_{0} / \tilde{J}>1.46$ while a Kondo phase is obtained for large $J_{K} / \tilde{J}$ values.

However, the calculated spin glass freezing temperature $\left(T_{f}\right)$ is lower than the Curie temperature $\left(T_{c}\right)$ in this highly frustrated model. Even the transition temperature to the mixed phase is always below the onset of the ferromagnetic order at $T_{c}$. Thus, our previous model [5] gives a ferromagnetic transition temperature $\left(T_{c}\right)$ above the spin glass transition temperature $\left(T_{f}\right)$, in contrast with the experimental situation of $C e N i_{1-x} C u_{x}$ [8, 9, 10, 11] alloys where the ferromagnetic phase is always the lowest one. That would be a clear indication that Gaussian distributed random couplings as in the Sherrington-Kirkpatrick (SK) model [19] is not adequate to describe the frustration present in that alloy.

One important point in the set of experimental works has been to clarify 
the effect of the disorder in the $C e N i_{1-x} C u_{x}$, 8, 9, 10, 11]. When $C u$ is randomly replaced by $N i$ in that alloy, not only the cell volume is modified, but also the number of conduction electrons which makes the competition between the RKKY and Kondo effect complex for that particular alloy [11. As a consequence, one localized spin at any site can be subject to a set of effective local magnetic fields, which results in the complicated combination of states previously cited.

In the Mattis model [15, which has been proposed as a solvable model to the spin glass problem, the bonds joining the localized spins have been defined as separable random variables $\xi_{i}$. This model could allow to gain some insight about the local effects of disorder as long as it would be possible to construct local applied fields dependent on the random variable $\xi_{i}$. Unfortunately, at zero magnetic field, a gauge transformation of the Ising spins classical variables leads Mattis model free energy to behave rather as the usual ferromagnet [16. 17. Therefore, this model is trivially disordered in the sense that it is unable to produce the essential component of the spin glass which is frustration. Nevertheless, the generalization of the Mattis model [18, 20] has proved to be an interesting alternative. In this model the coupling between spins are given by

$$
J_{i j}=\frac{1}{N} \sum_{\mu \nu} J_{\mu \nu} \xi_{i}^{\mu} \xi_{j}^{\nu}
$$

where $\xi_{i}^{\mu}= \pm 1(\mu=1,2, \ldots, p ; i=1,2, \ldots, N)$ are independent random distributed variables. For the classical Ising model, if $\mu=\nu=1$, the original Mattis model [15] is recovered. However, if $J_{\mu \nu}=J \delta_{\mu \nu}$ and $\mathrm{p}=\mathrm{N}$ with the $N^{2}$ random variables $\xi_{i}^{\mu}$ having mean zero and variance one, in the limit of $\mathrm{N}$ large, $J_{i j}$ tends to a Gaussian variable with mean zero and variance $N^{-1 / 2} J$ as in the SK model [19. Therefore, we can consider this model as an interpolation between ferromagnetism and highly disordered spin glass 22 .

An important particularity of this model (see Eq. (10) is $J_{\mu \nu}=J \delta_{\mu \nu}$ which has been used in a different context, i. e. the statistical mechanics theory of complex systems [22] using classical Ising spins. In this problem, randomness effects can be better understood at $T=0$ temperature when the local field applied in a particular spin given by $h_{i}=\sum_{i \neq j} J_{i j} S_{j}$ is analysed [21]. In the state $S_{i}=\xi_{i}^{1}$ (choosing $J=1$ ), the local field becomes $h_{i}=\xi_{i}^{1}\left(1+\delta_{i}\right)$, where $\delta_{i}$ is a random variable with variance $\left\langle\delta_{i}^{2}>_{\xi}=\frac{p-1}{N}\right.$. Two situations can be identified when $N \rightarrow \infty$ ( $N$ is the number of sites). If $p$ is finite, the spin is perfectly aligned with $\xi_{i}^{1}$. However, if $p$ increases linearly with $N(p=a N)$, the term $\delta_{i}$ can become important and the alignment can be destroyed. This random component of the local field can be a source of frustration and, in that sense, the ratio $\sqrt{N / p}$ is the analog of $J_{0} / \tilde{J}[22$. When temperature is turned over, there is an additional mechanism to avoid the aligning.

Actually, the thermodynamics of the generalized classical Mattis model can be described by a mean field theory [22, 23, 24, 21] in terms of the parameter $a=p / N$. For a particular value called $a_{c}$, two clearly distinct regimes can be identified. When $a>a_{c}$, the frustration is dominant below a certain temperature $T_{f}$ leading the problem to a spin glass behavior. Nevertheless, when $0<a<a_{c}$, a much more complex scenario can appear depending on the temperature. For instance, it can be found as solution not only a spin glass phase, but also Mattis states (which corresponds to the stable aligning between $\xi$ 's and 
spins) with a first order transition between them. These Mattis states have the same thermodynamics as the ferromagnetic phase [22, 23, 24, 21.

The mean field description of the previous situation introduces the order parameter $m^{\mu}=\frac{1}{N} \sum_{i} \xi_{i}^{\mu}<S_{i}>$ which gives a measure of the difference between the configurations of the set $\left\{\xi_{i}^{\mu}\right\}$ and the spins. In that approach, one particular solution can be, for example, only $m^{1}$ with a possible non zero value, while the remaining $m^{\mu}(\mu \neq 1)$ are of order $1 / \sqrt{N}$. This choice corresponds to the situation where the spins can be perfectly aligned only with $\xi_{i}^{1}$. Nevertheless, these $m^{\mu}$ 's $(\mu \neq 1)$ still have a role in the problem yelding a possible spin glass solution in the problem depending on the temperature and, particularly, on the parameter $a$. Therefore, this approach would be mathematically convenient to apply in a problem where we would be interested in getting control about the degree of frustration.

In this work, we consider the Kondo lattice model with a random intersite interaction between the localized spins where the coupling $J_{i j}$ is given by Eq. (11). That would allow us to investigate the competition between Kondo effect and magnetism combining the approach of Ref. [4] with Ref. [23]. Therefore, the degree of frustration $a=p / N$ is a new parameter which together with $J_{K} / J$ ( $J_{K}$ is the strength of the intra-site Kondo coupling) constitute the parameter space where the solutions can be located. In the Ref. 4, the solutions for the order parameters have been found only in the limit of strong frustration (the random Gaussian coupling) which corresponds to the $a>>a_{c}$ situation. We will show a more complex situation in the limit of weak frustration. For small $J_{K} / J$, there is an intermediate spin glass phase between paramagnetism and the region where there are Mattis states which corresponds to the ferromagnetism. Furthermore, the transition from the spin glass to the ferromagnetism is first order. Therefore, there is a large region in temperature where the ferromagnetic solution is thermodynamically metastable. It becomes stable at very low temperature. For large $J_{K} / J$, we get a Kondo state, as already discussed in Ref. [4.

It is not obvious that the properties of the classical Mattis model and its generalization can be extended to the quantum version of these models. However, it has been shown that the long range quantum Mattis model has the same qualitative behavior of his classical counterpart [25. Moreover, in the present fermionic spin glass mean field approach with the static approximation, which is reliable at high temperature, the spin variables have the essential features of classical ones [26, 27].

It should be remarked that the extension of the method given in Ref. 23 to the present fermionic problem is not straightforward. In fact, the model developed here is different from that one introduced in Refs. [5, 4, 6]. Indeed, both models study the competition between Kondo effect and spin glass, with eventually an additional magnetic phase. But, the description of the spin glass onset is different in the two cases. In the present one, the parameter $a$ can tune the spin glass or the ferromagnetic component of the long range internal field which has a completely different dependence on the spin glass order parameter as compared with Refs. [5, 4, 6]. Actually, for large values of the parameter $a$, the Gaussian distribution of spin couplings is recovered. That corresponds to the strong frustration situation studied in Ref. 4. Thus, the theoretical description is not simple, but the present model is able to give a more local description of the problem. Finally, as it will be discussed later on, we will obtain a ferromagnetic 
phase below the spin glass solution in terms of temperature, in contrast to the results of the previous model and in improved agreement with the experimental phase diagram of $C e N i_{1-x} C u_{x}$ alloys.

On the other hand, we can say that the experimental situation of $C e N i_{1-x} C u_{x}$ or similar alloys are also very complex. In fact, the so-called spin glass phase is more exactly a "cluster spin glass" which tends to a real ferromagnetic order by a percolative process when temperature decreases.

The outline of the paper is the following. In section II, the model is introduced and developed in order to get the free energy with the relevant order parameters for the problem. The results obtained are presented and discussed in section III, and we finished it up with a conclusion in the last section.

\section{General Formulation}

The model is the Kondo lattice used previously to study the competition between spin glass and Kondo effect [4. The Hamiltonian is given by

$$
H=\sum_{k, \sigma} \epsilon_{k} n_{k \sigma}^{c}+\sum_{i, \sigma} \epsilon_{0} n_{i \sigma}^{f}+H_{S G}+J_{K} \sum_{i}\left[\hat{S}_{f, i}^{+} \hat{s}_{i}^{-}+\hat{S}_{f, i}^{-} \hat{s}_{i}^{+}\right]
$$

where the sum is over the $N$ sites of a lattice.

The term $H_{S G}$ corresponds to the intersite interaction between localized spins, thus

$$
H_{S G}=\sum_{i, j} J_{i j} \hat{S}_{f i}^{z} \hat{S}_{f j}^{z}
$$

The random coupling $J_{i j}$ present in the previous equation is given by Eq. (11) with $J_{\mu \nu}=\frac{J}{2} \delta_{\mu \nu}$ [22], where $\xi_{i}^{\mu}= \pm 1$ are random independent variables which follow the distribution:

$$
P\left(\xi_{j}^{\mu}\right)=1 / 2 \delta_{\xi_{j}^{\mu},+1}+1 / 2 \delta_{\xi_{j}^{\mu},-1} .
$$

The spin operators in Eq. (2) are defined (see Refs. [5, 4, 6]) as bilinear combinations of the creation and destruction operators for localized (conduction) fermions $f_{i \uparrow}^{\dagger}, f_{i \downarrow}\left(d_{i \uparrow}^{\dagger}, d_{i \downarrow}\right)$ with the spin projection $\sigma=\uparrow$ or $\downarrow: \hat{S}_{f i}^{+}=f_{i \uparrow}^{\dagger} f_{i \downarrow}$; $\hat{S}_{f i}^{-}=f_{i \downarrow}^{\dagger} f_{i \uparrow} ; \hat{s}_{c i}^{+}=d_{i \uparrow}^{\dagger} d_{i \downarrow} ; \hat{s}_{c i}^{-}=d_{i \downarrow}^{\dagger} d_{i \uparrow}$;

$$
\hat{S}_{f i}^{z}=\frac{1}{2}\left[f_{i \uparrow}^{\dagger} f_{i \uparrow}-f_{i \downarrow}^{\dagger} f_{i \downarrow}\right] .
$$

The chemical potential for the localized and conduction bands are $\mu_{f}$ and $\mu_{c}$, respectively. As it has been done in Ref. [5, 4] 6], the energy $\epsilon_{o}$ is referred to $\mu_{f}$ and $\epsilon_{k}$ is referred to $\mu_{c}$.

In the functional integral formalism, the partition function is expressed using anticommuting Grassmann variables $\varphi_{i \sigma}(\tau)$ (related to conduction electrons) and $\psi_{i \sigma}(\tau)$ (related to the localized electrons) [4] as

$$
Z=\int D\left(\psi^{*} \psi\right) D\left(\varphi^{*} \varphi\right) \exp \left[A_{S G}+A_{K}+A_{0}\left(\psi^{*}, \psi\right)+A_{0}\left(\varphi^{*}, \varphi\right)\right]
$$


where in the static approximation [4]

$$
\begin{array}{r}
A_{0}\left(\psi^{*}, \psi\right)=\sum_{i j \sigma} \sum_{\omega} \psi_{i \sigma}^{*}(\omega)\left[i \omega-\beta \varepsilon_{0}\right] \delta_{i j} \psi_{j \sigma}(\omega), \\
A_{0}\left(\varphi^{*}, \varphi\right)=\sum_{i j, \sigma, \omega} \varphi_{i \sigma}^{*}(\omega)\left[\left(i \omega-\beta \varepsilon_{k}\right) \delta_{i j}-t_{i j}\right] \varphi_{j \sigma}(\omega), \\
A_{K}^{s t a t} \approx \frac{J_{K}}{N} \sum_{i \sigma} \sum_{\omega}\left[\varphi_{i-\sigma}^{*}(\omega) \psi_{i-\sigma}(\omega)\right] \sum_{j \sigma} \sum_{\omega^{\prime}}\left[\psi_{j \sigma}^{*}\left(\omega^{\prime}\right) \varphi_{j \sigma}\left(\omega^{\prime}\right)\right], \\
A_{S G}^{s t a t}=\sum_{i j} J_{i j} S_{i} S_{j} .
\end{array}
$$

with

$$
S_{i}=\frac{1}{2} \sum_{\sigma= \pm} \sum_{\omega} \sigma \psi_{i \sigma}^{\dagger}(\omega) \psi_{i \sigma}(\omega)
$$

In Eq. (11), the Matsubara's frequencies are given, as usual, by $\omega=(2 m+1) \pi$ with $m=0, \pm 1, \pm 2, \ldots$.

The problem is treated closely to the mean field approximation of Ref. [4. Therefore, the Kondo order parameter $\lambda_{\sigma}$ and its conjugate are introduced using the integral representation of the delta function as

$$
\begin{array}{r}
\delta\left(N \lambda_{\sigma}-\sum_{\omega} \sum_{i=1}^{N} \varphi_{i \sigma}^{*}(\omega) \psi_{i \sigma}(\omega)\right)=\int \prod_{\sigma} \frac{d v_{\sigma}}{2 \pi} \times \\
\exp \left\{i \sum_{\sigma} v_{\sigma}\left[N \lambda_{\sigma}^{*}-\sum_{\omega} \sum_{i=1}^{N} \varphi_{i \sigma}^{*}(\omega) \psi_{i \sigma}(\omega)\right]\right\}
\end{array}
$$

where the presence of the order parameter $\lambda_{\sigma}$ is related to the formation of $d-f$ singlet throughout the whole lattice. This mean field order parameter is presently recognized to provide good description of the Kondo effect on each site 29

Therefore, the resulting partition function becomes:

$$
Z=\int \prod_{\sigma} d \lambda_{\sigma}^{\dagger} d \lambda_{\sigma} \exp \left[-N \beta J_{K} \sum_{\sigma} \lambda_{\sigma}^{\dagger} \lambda_{\sigma}\right] Z_{\text {stat }}
$$

where

$$
\begin{gathered}
Z_{\text {stat }}=\int D\left(\psi^{*} \psi\right) D\left(\varphi^{*} \varphi\right) \exp \left\{A_{0}\left(\psi^{*}, \psi\right)+A_{S G}^{s t a t}+A_{0}\left(\varphi^{*}, \varphi\right)\right. \\
\left.+\beta J_{K} \sum_{\sigma}\left[\lambda_{-\sigma}^{\dagger} \sum_{j, \omega} \varphi_{j \sigma}^{\dagger}(\omega) \psi_{j \sigma}(\omega)+\lambda_{\sigma} \sum_{j, \omega} \psi_{j \sigma}^{\dagger}(\omega) \varphi_{j \sigma}(\omega)\right]\right\}
\end{gathered}
$$

In fact, in this work the Kondo order parameter is considered $\lambda_{\sigma}=\lambda$ [4, 5, 6].

The integration over the Grassmann fields $\varphi^{*}$ and $\varphi$ in Eq. (14) can be performed, which results in:

$$
\frac{Z_{\text {stat }}}{Z_{0}}=\int D\left(\psi^{*} \psi\right) \exp \left[A_{0}^{\text {eff }}+A_{S G}^{\text {stat }}\right]
$$


where

$$
A_{0}^{e f f}=\sum_{i j \sigma} \sum_{\omega} \psi_{i \sigma}^{*}(\omega) g_{i j}^{-1}(\omega) \psi_{j \sigma}(\omega)
$$

with

$$
g_{i j}^{-1}(\omega)=\left(i \omega-\beta \epsilon_{0}\right) \delta_{i j}-\beta^{2} J_{K}^{2} \lambda^{*} \lambda \gamma_{i j}(\omega) .
$$

The Fourier transform of Green's function $\gamma_{i j}(\omega)$ in Eq. (17) is

$$
\gamma_{k}(\omega)=\frac{1}{i \omega-\beta \varepsilon_{0}-\beta \varepsilon_{k}}
$$

In order to introduce the proper set of order parameters in our problem 23, the action $A_{S G}^{\text {stat }}$ in Eq. (10) is written using Eq. (11) to give

$$
A_{S G}^{\text {stat }}=\frac{\beta J}{2 N} \sum_{\mu=1}^{p}\left(\sum_{i} \xi_{i}^{\mu} S_{i}\right)^{2}-\frac{\beta J p}{2 N} \sum_{i}\left(S_{i}\right)^{2} .
$$

where $S_{i}$ has been defined in Eq. (11).

The free energy can be obtained following the replica method,

$$
\beta f=2 \beta J_{k} \lambda^{*} \lambda-\lim _{n \rightarrow 0} \frac{1}{N n}\left(\left\langle\left\langle Z_{\text {stat }}^{n}\right\rangle\right\rangle_{\xi}-1\right),
$$

where $\langle\langle\cdots\rangle\rangle_{\xi}$ is the averaged over $\xi$ 's.

The fundamental issue consists in the evaluation of the quadratic form present in the first term of $A_{S G}^{\text {stat }}$. First, the sum over $\mu$ is separated in two parts [23]: $\sum_{\mu=1}^{p}=\sum_{\mu=s}^{p}+\sum_{\nu=1}^{s-1}$.

It is possible to linearize the problem introducing $n \times p$ auxiliary fields $m_{\alpha}^{\mu}$ and $m_{\alpha}^{\nu}$ ( $\alpha$ is a replica index) which correspond to the parameter discussed in the previous section. Therefore,

$$
\begin{array}{r}
\exp \left(A_{S G}^{\text {stat }}\right)=\exp \left[-\frac{\beta J p}{2 N} \sum_{\alpha=1}^{n} \sum_{i=1}^{N}\left(S_{i}^{\alpha}\right)^{2}\right] \times \\
\int_{-\infty}^{\infty} D m_{\alpha}^{\mu} \exp \left\{\beta J N \sum_{\nu=1}^{s-1} \sum_{\alpha}\left[-\frac{1}{2}\left(m_{\alpha}^{\nu}\right)^{2}+\frac{1}{N} \sum_{i} \xi_{i}^{\nu} S_{i}^{\alpha} m_{\alpha}^{\nu}\right]\right\} \int_{-\infty}^{\infty} D m_{\alpha}^{\nu} \times \\
\exp \left\{\beta J N \sum_{\mu=s}^{p} \sum_{\alpha}\left[-\frac{1}{2}\left(m_{\alpha}^{\mu}\right)^{2}+\frac{1}{N} \sum_{i} \xi_{i}^{\mu} S_{i}^{\alpha} m_{\alpha}^{\mu}\right]\right\} .
\end{array}
$$

where $D m_{\alpha}^{\mu(\nu)}=\prod_{\mu(\nu)} \prod_{\alpha} d m_{\alpha}^{\mu(\nu)} / \sqrt{2 \pi}$.

In this work, the structure of solutions for auxiliary fields $m_{\alpha}^{\mu}$ 's and $m_{\alpha}^{\nu}$ 's is the same as in Ref. 23. We assume that the relevant contributions come from $m_{\alpha}^{\nu}$ which are order unity while $m_{\alpha}^{\mu}$ is of order $1 / \sqrt{N}$. Therefore, the average over the $p-s$ independent random variables $\xi_{i}^{\mu}$ can be done using Eq. (4) which results in:

$\left\langle\left\langle\exp \left[\beta J \sum_{\mu=s}^{p} \sum_{\alpha}\left(\sum_{i} \xi_{i}^{\mu} S_{i}^{\alpha}\right) m_{\alpha}^{\mu}\right]\right\rangle\right\rangle_{\xi}=\exp \left[\sum_{i} \sum_{\mu=s}^{p} \ln \left(\cosh \left(\beta J \sum_{\alpha} S_{i}^{\alpha} m_{\alpha}^{\mu}\right)\right)\right]$. 
The argument of the exponential in the right hand side of Eq. (22) can be expanded up to second order in $m_{\alpha}^{\mu}$ The result is a quadratic term of the spins variables $S_{i}^{\alpha}$ in the last exponential of Eq. (21). This term can be linearized by introducing the spin glass order parameter $q_{\alpha \beta}$ using the integral representation of the delta function as we have done with the Kondo order parameter, so

$$
1=\int_{-\infty}^{\infty} \prod_{\alpha \beta} \delta\left(q_{\alpha \beta}-\frac{1}{N} \sum_{i} S_{i}^{\alpha} S_{i}^{\beta}\right) .
$$

where $q_{\alpha \beta}$ is equivalent to the usual spin glass order parameter introduced in the classical SK model [19] which gives the transition to spin glass phase when $m_{\alpha}^{\mu}=0$.

Therefore, after rescaling $m_{\alpha}^{\mu} \rightarrow m_{\alpha}^{\mu} / \sqrt{N}$, the exponential involving $m_{\alpha}^{\mu}$ in Eq. (21) can be written as:

$$
\begin{array}{r}
\exp \left\{\beta J N \sum_{\mu=s}^{p} \sum_{\alpha}\left[-\frac{1}{2}\left(m_{\alpha}^{\mu}\right)^{2}+\frac{1}{N}\left(\sum_{i} \xi_{i}^{\mu} S_{i}^{\alpha}\right) m_{\alpha}^{\mu}\right]\right\} \\
=\frac{1}{2 \pi} \int_{-\infty}^{\infty}\left(\prod_{\alpha \beta} d q_{\alpha \beta} d \bar{r}_{\alpha \beta}\right) \exp \left\{-\frac{\beta J}{2} \sum_{\mu=s}^{p} m_{\alpha}^{\mu} \Lambda_{\alpha \beta} m_{\beta}^{\mu}\right. \\
\left.i \sum_{\alpha \beta} \bar{r}_{\alpha \beta}\left(q_{\alpha \beta}-\frac{1}{N} \sum_{i} S_{i}^{\alpha} S_{i}^{\beta}\right)\right\}
\end{array}
$$

where the matrix element

$$
\Lambda_{\alpha \beta}=\delta_{\alpha \beta}-\beta J q_{\alpha \beta} .
$$

Introducing Eqs. (24) and (25) into Eq. (21), the $m_{\alpha}^{\mu}$ fields can be integrated to give:

$$
\begin{array}{r}
\left\langle\left\langle\exp A_{S G}^{\text {stat }}\right\rangle\right\rangle_{\xi}=\exp \left[-\frac{\beta J p}{2 N} \sum_{\alpha=1}^{n} \sum_{i=1}^{N}\left(S_{i}^{\alpha}\right)^{2}\right]\left\langle\left\langle\int_{-\infty}^{+\infty} D m_{\alpha}^{\nu}\right.\right. \\
\left.\left.\exp \left\{\beta J N \sum_{\nu=1}^{s-1} \sum_{\alpha}\left[-\frac{1}{2}\left(m_{\alpha}^{\nu}\right)^{2}+\frac{1}{N}\left(\sum_{i} \xi_{i}^{\nu} S_{i}^{\alpha}\right) m_{\alpha}^{\nu}\right]\right\}\right\rangle\right\rangle_{\xi} \int_{-\infty}^{+\infty}\left(\prod_{\alpha \beta} \frac{d q_{\alpha \beta} \bar{r}_{\alpha \beta}}{2 \pi}\right) \\
\left.\exp \left\{i \sum_{\alpha \beta} \bar{r}_{\alpha \beta}\left(q_{\alpha \beta}-\frac{1}{N} \sum_{i=1}^{N} S_{\alpha}^{i} S_{\beta}^{i}\right)-\frac{1}{2}(p-s) \operatorname{Tr} \ln \underline{\underline{\Lambda}}\right)\right\} .
\end{array}
$$

Therefore, the averaged partition function (see Eq. (20) is obtained from Equations (1517) and (26) as

$$
\begin{array}{r}
\langle\langle Z\rangle\rangle_{\xi}=\int_{-\infty}^{+\infty}\left(\prod_{\alpha \nu} d m_{\alpha}^{\nu}\right) \int_{-\infty}^{+\infty}\left(\prod_{\alpha \beta} \frac{d q_{\alpha \beta} \bar{r}_{\alpha \beta}}{2 \pi}\right) \times \\
\exp \left\{-\beta N\left[\frac{J}{2} \sum_{\alpha}\left(m_{\alpha}^{\nu}\right)^{2}+\frac{p-s}{2 N \beta} \operatorname{Tr} \ln \underline{\underline{\Lambda}}-\right.\right. \\
\left.\left.\frac{i}{N \beta} \sum_{\alpha} \bar{r}_{\alpha \alpha} q_{\alpha \alpha}-\frac{i}{N \beta} \sum_{\alpha \neq \beta} \bar{r}_{\alpha \beta} q_{\alpha \beta}\right]\right\}\langle\langle\Omega\rangle\rangle_{\xi}
\end{array}
$$


where

$$
\begin{array}{r}
\Omega=\int D\left(\psi^{*} \psi\right) \exp \left\{-i \sum_{\alpha \neq \beta} \bar{r}_{\alpha \beta}\left(\frac{1}{N} \sum_{i} S_{i}^{\alpha} S_{i}^{\beta}\right)-\right. \\
\sum_{\alpha}\left(\frac{\beta J p}{2}+i \bar{r}_{\alpha \alpha}\right) \frac{1}{N} \sum_{i}\left(S_{i}^{\alpha}\right)^{2}+\beta J\left(\sum_{i} \xi_{i}^{\nu} S_{i}^{\alpha}\right) m_{\alpha}^{\nu}+ \\
\left.\sum_{i j} \sum_{\sigma \alpha} \sum_{\omega} \psi_{i \sigma \alpha}^{*}(\omega) g_{i j}^{-1}(\omega) \psi_{j \sigma \alpha}(\omega)\right\}
\end{array}
$$

The free energy (see Eq. 20) is evaluated at the saddle point and is given by the condition that the first derivatives of integrating variables are zero. Therefore, for instance, we have

$$
-i \bar{r}_{\alpha \alpha}=\frac{\beta^{2} J^{2}}{2}\left\langle\sum_{\nu}\left(m_{\alpha}^{\nu}\right)^{2}\right\rangle=\frac{\beta^{2} J^{2}}{2} p r_{\alpha \alpha}
$$

and

$$
-i \bar{r}_{\alpha \beta}=\frac{\beta^{2} J^{2}}{2}\left\langle\sum_{\nu}\left(m_{\alpha}^{\nu} m_{\beta}^{\nu}\right)\right\rangle=\frac{\beta^{2} J^{2}}{2} p r_{\alpha \beta} ; \quad \alpha \neq \beta .
$$

The parameter $m_{\alpha}^{\nu}$ has been found as in the section I. The problem is treated assuming the replica symmetric ansatz, therefore the order parameters are $q_{\alpha \beta}=$ $q, q_{\alpha \alpha}=\bar{q}, r_{\alpha \alpha}=\bar{r}, r_{\alpha \beta}=r$ and $m_{\alpha}^{\nu}=m^{\nu}$. On the other hand, the trace of the matrix $\underline{\underline{\Lambda}}$ is obtained in terms of its eigenvalues [22] (see Eq. 25). In consequence, the free energy can be written as

$$
\begin{array}{r}
\beta f=2 \beta J_{K}|\lambda|^{2}+\frac{\beta J}{2} \sum_{\nu}\left(m^{\nu}\right)^{2}-\frac{a}{2}\left(\frac{\beta J q}{1-\beta J(\bar{q}-q)}\right) \\
+\frac{a}{2} \ln [1-\beta J(\bar{q}-q)]+\frac{\beta^{2} J^{2} a}{2} \bar{r} \bar{q}-\frac{\beta^{2} J^{2} a}{2} r q- \\
\lim _{n \rightarrow 0} \frac{1}{n N} \ln \left[\left\langle\left\langle\Omega\left(r, \bar{r}, m^{\nu},|\lambda|\right)\right\rangle\right\rangle_{\xi}\right]
\end{array}
$$

where $a=p / N$.

The sum over replica index produces quadratic forms into the function $\Omega\left(r, \bar{r}, m^{\nu},|\lambda|\right)$. This term can be linearized by a Hubbard-Stratonovich transformation [4, 5, 6] where new auxiliary fields are introduced in the problem. Therefore, we have:

$$
\begin{array}{r}
\Omega\left(r, \bar{r}, m^{\nu},|\lambda|\right)= \\
\int_{-\infty}^{+\infty} \prod_{j=1}^{N} D z_{j} \int_{-\infty}^{+\infty} \prod_{j=1}^{N} \prod_{\alpha=1}^{n} D w_{j}^{\alpha} \int D\left(\psi^{*} \psi\right) \\
\exp \left[\sum_{i j} \sum_{\alpha, \sigma} \sum_{\omega} \psi_{i \sigma \alpha}^{*}(\omega) G_{i j}^{-1}(\omega) \psi_{j \sigma \alpha}(\omega)\right]
\end{array}
$$

with $D z=\frac{d z}{\sqrt{2 \pi}} e^{-z^{2} / 2}$ and

$$
G_{i j}^{-1}(\omega)=g_{i j}^{-1}(\omega)-\sigma\left(\bar{h}_{i}^{\alpha}(r, \bar{r})+\beta \sum_{\nu} \xi_{i}^{\nu} m^{\nu}\right) \delta_{i j}
$$


where $g_{i j}^{-1}(\omega)$ is given by Eq. (16) and the local spin glass component of the internal field

$$
\bar{h}_{i}^{\alpha}(r, \bar{r})=\sqrt{\beta J a[\beta J(\bar{r}-r)-1]} w_{i}^{\alpha}+\beta J \sqrt{a r} z_{i} .
$$

The functional integral in Eq. (32) can be performed [5, 4, 6], so we get

$$
\begin{array}{r}
\Omega\left(r, \bar{r}, m^{\nu},|\lambda|\right)=\int_{-\infty}^{+\infty}\left(\prod_{j=1}^{N} D z_{j}\right) \\
\int_{-\infty}^{+\infty}\left(\prod_{j=1}^{N} \prod_{\alpha=1}^{n} D w_{j}^{\alpha}\right) \exp \left[\sum_{\omega} \sum_{\alpha, \sigma} \ln \left[\operatorname{det} G_{i j}^{-1}(\omega)\right]\right]
\end{array}
$$

The local field $\bar{h}_{i}^{\alpha}(r, \bar{r})$ applied in the $n$ replicated lattices is, in fact, associated with the replica diagonal and non-diagonal spin glass order parameters. The presence of the local field is the fundamental technical issues which must be solved in order to proceed the calculations. Therefore, we consider the following decoupling [5, 4, 6]:

$$
\ln \left[\operatorname{det} G_{i j}^{-1}(\omega)\right] \approx \frac{1}{N} \sum_{i} \ln \left[\operatorname{det} \Gamma_{\delta \nu \sigma}\left(\omega, \bar{h}_{i}^{\alpha}(r, \bar{r}), \xi_{i}^{\nu}\right)\right]
$$

which means that a constant field is applied in a fictitious Kondo lattice, so the problem can be solvable by a Fourier transform. The particular form of the decoupling is also useful because it allows the use of self-averaging property $\frac{1}{N} \sum_{i} f\left(\xi_{i}\right)=\langle\langle f(\xi)\rangle\rangle_{\xi}[22$, which is valid in thermodynamic limit for finite $s-1$, the upper value of $\nu$. Actually, from now on, it is assumed that $s=2$. The self-averaging in Eq. (36) allows us to drop the site index $i$ in Eq. (35).

The resulting sum over $k$ in Fourier transformed $\Gamma_{k, \sigma}\left(\omega, \bar{h}^{\alpha}(r, \bar{r}), \xi\right)$ can be replaced by an integral using a constant density of states for the conduction electrons $\rho(\epsilon)=\frac{1}{2 D}$ for $-D<\epsilon<D$. On the other hand, the sum over the Matsubara's frequencies in Eq. (35) can be performed following closely the procedure given in Refs. 5, 4, 6]. Finally, the free energy can be found as

$$
\begin{array}{r}
\beta f=2 \beta J_{K}|\lambda|^{2}+\frac{\beta J}{2}\left(m^{1}\right)^{2}+\frac{a}{2} \ln [1-\beta J(\bar{q}-q)]-\frac{a}{2} \frac{\beta J q}{1-\beta J(\bar{q}-q)}+ \\
\frac{\beta^{2} J^{2} a}{2} \bar{r} \bar{q}-\frac{\beta^{2} J^{2} a}{2} r q-\int_{-\infty}^{+\infty} D z\left\langle\left\langle\operatorname { l n } \left\{\int _ { - \infty } ^ { + \infty } D w \operatorname { e x p } \left[\frac{1}{\beta D} \int_{-\beta D}^{+\beta D} d x \times\right.\right.\right.\right. \\
\left.\left.\left.\ln \left[2 \cosh \left(\frac{x+h(r, \bar{r}, \xi)}{2}\right)\right]+2 \cosh \left(\sqrt{\Delta(r, \bar{r}, \xi)^{2}+\left(\beta J_{K}|\lambda|\right)^{2}}\right]\right\}\right\rangle\right\rangle_{\xi}
\end{array}
$$

where

$$
\Delta(r, \bar{r}, \xi)=\frac{x-h(r, \bar{r}, \xi)}{2}
$$

and

$$
h(r, \bar{r}, \xi)=\sqrt{\beta J a[\beta J(\bar{r}-r)-1]} w+\beta J \sqrt{a r} z+\beta J m^{1} \xi
$$

is a long range internal field composed by two parts, a spin glass one already introduced in Eq. (34) and other one associated with the order parameter 


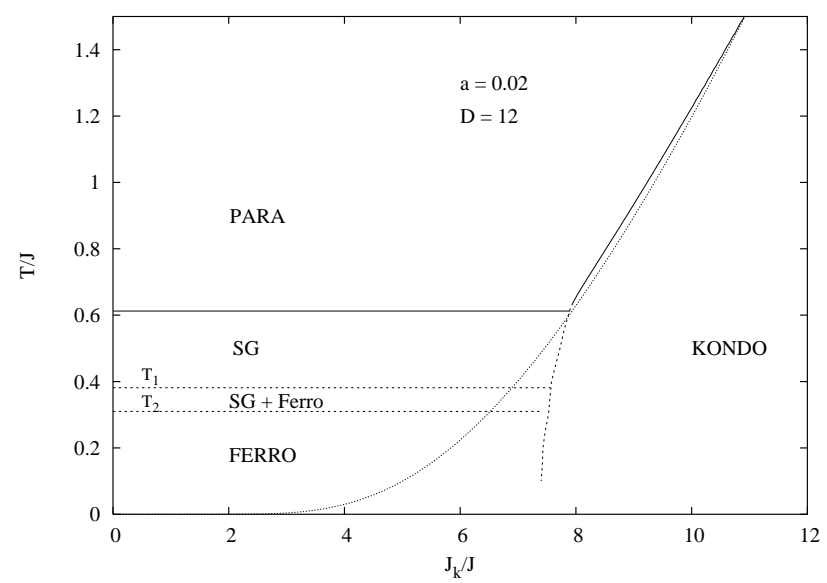

Figure 1: The phase diagram with $T / J$ versus $J_{K} / J$ for $a=0.02$ showing the phases SG (spin glass), FERRO (ferromagnetism) and KONDO (the Kondo state). In the region SG+Ferro, there is coexistence between SG and FERRO. The dotted line means the "pure" Kondo temperature.

$m^{1}$. This result can be compared with Ref. [5]. In that work, a random Gaussian inter-site coupling among the localized spins has been used. The equivalent internal field found there can be also decomposed in two parts: a ferromagnetic one, and a spin glass term associated with $q$ and the static susceptibility $\chi=\beta(\bar{q}-q)$. However, the dependence of the internal field with $q$ and $\bar{q}$ is entirely distinct in the present work as it will be shown below.

The coupled set of equations for the order parameters can be found from Equation (37) using the saddle point conditions. In particular, there is a relationship between the following order parameters:

$$
\begin{gathered}
r=\frac{q}{[1-\beta J(\bar{q}-q)]^{2}}, \\
r-\bar{r}=\frac{1}{\beta J} \frac{1}{[1-\beta J(\bar{q}-q)]} .
\end{gathered}
$$

If we replace Eqs. (40 41) in Eqs. (3739), the theory becomes explicitly dependent on the $q$ and $\bar{q}$. Therefore, the minimum set of order parameters to be solved in order to obtain a global phase diagram is the spin glass order parameters $q, \bar{q}$ (related to diagonal matrix $q_{\alpha \alpha}$ ), the Kondo order parameter $|\lambda|$ and the $m^{1}=m$. The average over $\xi$ in Eq. (37) can be now performed using the parity properties of the functions dependent on $z$ and $w$. Therefore, the dependence on $\xi$ can be dropped. The remaining set of coupled equations for the order parameters are given by the corresponding saddle point equations.

\section{Discussions}

In this paper, it has been investigated the competition between ferromagnetism and spin glass in a Kondo lattice model with a random coupling between 


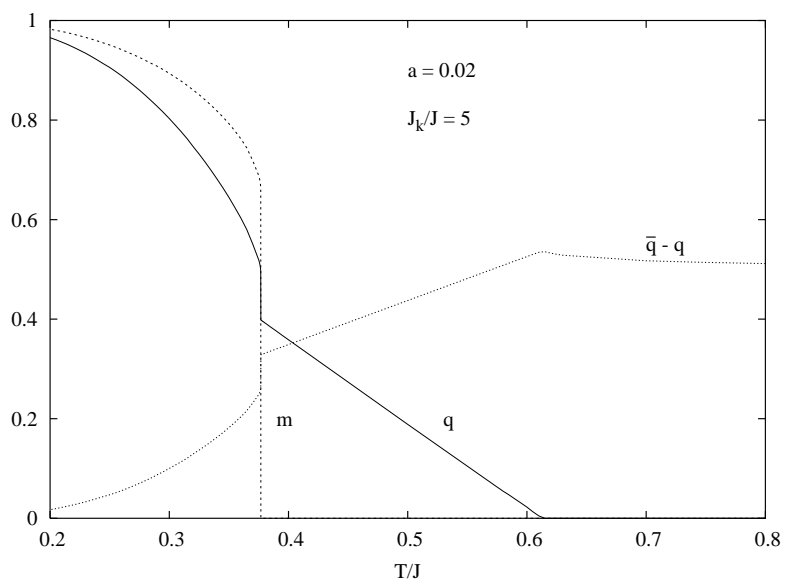

Figure 2: The behavior of the order parameters $q, \bar{q}$ and $m$ showing the second order and the first order transition for $J_{K}=5 \mathrm{~J}$.

localized spins. The coupling is constructed (see Eq. (1)) as a product of two random independent variables $\xi_{i}^{\mu}$ where $i=1 \ldots N$ (N number of sites) and $\mu=1 \ldots p$ which is a generalization of the Mattis model [15] for random magnetic systems. In the strongly frustrated limit $p \rightarrow \infty$ (when $N \rightarrow \infty$ and $p / N=1$ ), it is recovered a spin glass solution as S-K model 18. The problem is solved using functional integral formalism, the static aproximation and the replica ansatz. The order parameters are obtained by combining methods proposed in Refs. 4], 22] which allow us to introduce an aditional parameter $a=p / N$ to control the level of frustration.

The numerical solutions of the order parameters $q, \bar{q}, m$ and $|\lambda|$ (from now on $|\lambda|=\lambda$ ) give as solutions the following thermodynamic phases: (a) a spin glass where $q \neq 0$ with $\lambda=0$ and $m=0$; (b) a Kondo state with $\lambda \neq 0$ and $q=0$ and $m=0 ;$ (c) ferromagnetism which is given by the existence of the Mattis states described by $m \neq 0$ and $q \neq 0$ with $\lambda=0$. The solutions are displayed in diagrams $T / J$ versus $J_{K} / J$ for several values of $a$, where $T$ is the temperature, $J_{K}$ and $J$ are the strength of the intrasite Kondo coupling and the intersite random coupling between localized spins (see Eqs. (2)-(4)), respectively. Therefore, for a given $J_{K} / J$, it is possible to probe solutions for the order parameters equations $q, \bar{q}, m$ and $\lambda$ in several situations ranging from weak frustration to strong one just by varying the parameter $a$.

The physical origin of the phases discussed in the previous paragraph can be understood from the model introduced in Eqs. (2)-(3) in which there are two interactions. The first one is on site Kondo coupling while the second one is the disordered coupling between localized spins. Therefore, it is possible to identify several energy scales in the problem as $T, J_{K}$ and $J$. When temperature is high enough, there is only paramagnetism. As long as the Kondo energy scale becomes dominant in relation to the remaining ones, the emerging ordering is the complete screening of the localized spins in the whole lattice due to the Kondo effect. However, in a certain range of $J_{K} / J$, there are two possible magnetic orderings. In the spin glass one, the competing interactions between the spins can give rise to frustration in which there is a large number of degenerate states 


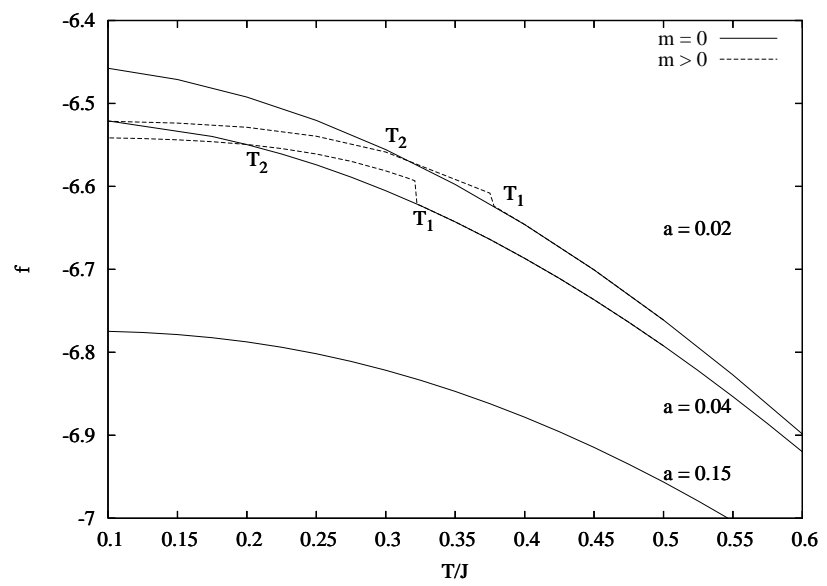

Figure 3: The free energy as a function of temperature for $a=0.02, a=0.04$, and $a=0.15$ showing the region (between $T_{1}$ and $T_{2}$ ) where there are multiple solutions for the order parameters.

for the spins configurations. Therefore, there is no long range order correlation among spins orientations due to the presence of frustration which leads the spins to be frozen in random orientations. In contrast, in the ferromagnetic regime, the Mattis states correspond to the situation in which the spins stabilize aligned with the $\xi_{i}$ 's due to the low frustration level. The prevalence of one or other regime discussed above depends on the parameter $a$ which controls the frustration level for the random coupling given in Eq. (1) as well as $T$.

It should be remarked that previous investigation, using the same model, has adopted the standard Gaussian random coupling [5, 4] (as the S-K model) for the intersite interaction between localized spins. Nevertheless, in this strongly frustrated approach $T_{c}>T_{f}\left(T_{c}\right.$ and $T_{f}$ are the Curie and freezing temperatures, respectively) in disagreement with experimental results for $\mathrm{CeNi} i_{1-x} C u_{x}$. Therefore, the motivation for the present work is to understand better the role of disorder as source of frustration in a Kondo lattice model and, for that reason to possibly address the experimental findings of $C e N i_{1-x} C u_{x}$ phase diagram.

In Fig. 1, the results for $a=0.02$ are presented. For high $T / J$ and small $J_{K} / J$, the numerical solutions display a paramagnetic (PARA) behavior with $q=0, m=0$ and $\lambda=0$. The solutions remain the same in this small $J_{K} / J$ region until $T \approx 0.61 J$, where $q$ starts to be continuously non-zero indicating a second order phase transition to a spin glass phase. The behavior of the order parameters as a function of the temperature is shown in Fig. 2. In particular, we can see that at the same temperature where $q \neq 0$, there is also a cusp in $\bar{\chi}=\chi / \beta=(\bar{q}-q)$ ( $\chi$ is static susceptibility). If the temperature is lowered, the results remain yielding a spin glass solution until $T \approx 0.38 \mathrm{~J}$. From that point, the parameters $m$ and $q$ become simultaneously different from zero similar to the ferromagnetic solution already found in Ref. [5]. However, in the present case there is an abrupt change in their behaviour indicating a first order transition. In fact, for $0.31 J \lesssim T \lesssim 0.38 J$, we have found metastable solutions with $m \neq 0$ and $q \neq 0$ which corresponds to the Mattis states. The emergence of these metastable Mattis states at temperature $T_{1}$ can be seen clearly just 


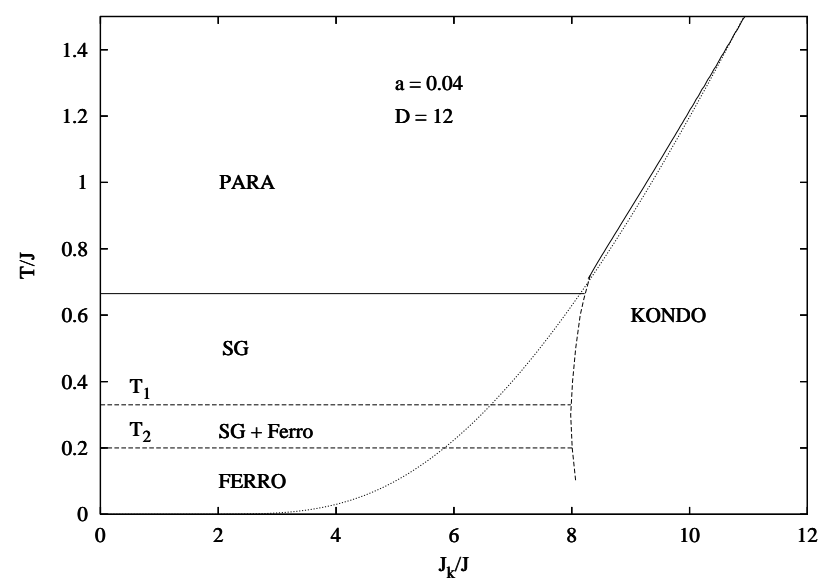

Figure 4: The phase diagram with $T / J$ versus $J_{K} / J$ for $a=0.04$ showing the phases SG (spin glass), FERRO (ferromagnetism) and KONDO (the Kondo state). In the region SG+Ferro, there is the coexistence between SG and FERRO. The dotted line means the "pure" Kondo temperature.

by following the free energy (see Fig. 3). In Fig. 1, the corresponding region has been named as SG + Ferro. Finally, at $T_{2}$, the spin glass solutions become unstable thermodynamically while the solution with $m \neq 0$ and $q \neq 0$ (the Mattis states) becomes stable.

When $J_{K} / J$ is enhanced (see Fig. 1), it is found a line transition $J_{K_{c}}(T / J)$ for the Kondo state. This kind of solution had already been found in Refs. [5, 4, 6. The nature of this line transition is complex. It is second order at high temperature changing to first order at low temperature. However, there are evidences indicating that this complexity could be nonphysical, in fact, related to the approximations made in the present approach [28.

If the parameter $a$ is increased (for instance $a=0.04$, in Fig. 4), we have, basically, a phase diagram displaying the same situation already shown in Fig. 1. However, the spin glass stability range is increased. Finally, for $a=0.15$, there is no more Mattis states as solution, the spin glass solution is entirely dominating for $J_{K}<J_{K c}(T)$ in which $J_{K c}(T)$ is the phase boundary of the Kondo state. In this limit, it is recovered the results obtained in Ref. 4.

The experimental results for $C e N i_{1-x} C u_{x}$ can be now addressed. The phase diagrams obtained with bulk methods [8] and $\mu$ SR technique [10] show that the chemical substitution of $N i$ produces in the alloy a complex interplay between Kondo effect and magnetism where, for example, the spin glass phase is always found at higher temperature than the ferromagnetic one (whatever the nature of the spin glass region is). Moreover, it also shows the increase of the freezing temperature with the decrease of $C u$ content in the alloy until $x \approx 0.4$. On the other hand, for $x \lesssim 0.2$ there is a considerable reduction of $C e$ localized magnetic moments due to the Kondo effect.

If it is assumed that the substitution of $C u$ by $N i$ can be related to the parameters $J_{K}$ and $a$, some important aspects of the experimental scenario can be reproduced, for example:

i) For $J_{K}<J_{K c}(T)$ and small $a$ (weak frustration). In this regime the mag- 


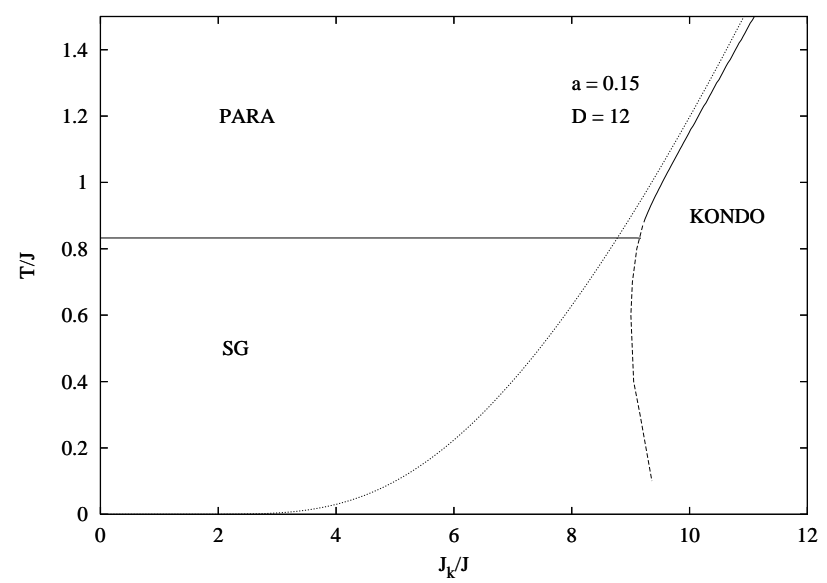

Figure 5: The phase diagram with $T / J$ versus $J_{K} / J$ showing the phase SG (spin glass). The dotted line means the "pure" Kondo temperature.

netic solutions are dominant with no trace of a Kondo solution. However, the combination of high temperature and the local complex randomness prevents any kind of stable alignement of the spins (represented by the order parameter $m)$. On the contrary, the solutions for the order parameter show a continous direct transitions from paramagnetism to spin glass. Eventually, at lower temperature, the randomness is not enough to keep avoiding any stable alignement of the spins. Therefore, the Mattis states start to appear first as metastable solutions until becoming the thermodynamical stable solutions of the problem. As consequence, in this weak frustration limit, the correct order for the transition temperatures is recovered as compared with the experimental situation when $0.8 \gtrsim x \gtrsim 0.4$, where $x$ is the content of $C u[9$.

As long as the level of frustration $a$ is increasing, the spin glass component of the internal field becomes dominant in a larger range of temperature. Therefore, it is obtained an enlargement of the spin glass region (see Figure 1, 4 and 5) which precedes the onset of the ferromagnetism. As a consequence, the freezing temperature $T_{f}$ is also increased, which resembles the experimental situation when $x \rightarrow 0.4$ [ .

ii) When $J_{K}>J_{K c}(T)$, the magnetic solutions disappear and the Kondo state appears as the unique solution in the problem for any value of $a$ which means that there is a complete screening of localized spins due to the Kondo effect as in the experimental results for the rich $N i$ region 9 .

However, there is still some disagreement related to the results obtained from $\mu \mathrm{Sr}$ spectroscopy [10] and specific heat [11] which suggests the presence of nanoclusters which are frozen at some temperature [1]. For lower temperatures, those clusters would percolate yielding a ferromagnetism [11] in a similar process proposed to explain manganites 30. Our results, instead, in the weak frustrated limit, indicate a continuous transition to a spin glass phase and, then, at lower temperature, the presence of a first order one with a coexistence region between spin glass and ferromagnetism (given by the Mattis states). Nevertheless, we believe that the present mean field theory is a clear improvement in the sense that it provides an effective mechanism which, at least, gives the correct ordering 
of the magnetic transition temperatures.

To conclude, in this work, it has been studied the spin glass-ferromagnetismKondo phase transitions in a Kondo lattice model with a random coupling $J_{i j}$ between the localized spins. The $J_{i j}$ is given as a product of random variables $\xi_{i}^{\mu}(\mu=1 \ldots p, i=1 \ldots N)$. This choice for $J_{i j}$ introduces a parameter $a=$ $p / N$ which allows us to control the degree of frustration. Thereby, the balance between the two parameters $J_{K} / J$ and $a$ controls the emergence of the different solutions in the problem. For small $a$ (weak frustration) and $J_{K} / J$, we have found that there is only the presence of spin glass and ferromagnetic solutions, and, particularly that the freezing temperature is higher than the transition temperature where ferromagnetic solutions are found in good agreement with experiment in $\mathrm{Ce}(\mathrm{Ni}, \mathrm{Cu})$ alloys. For large $J_{K} / J$, there is only a Kondo state, whatever the value of $a$ is. The results obtained here are interesting for the study of the role of disorder, which seems to be better described by taking into account an average of discrete values $\xi$ 's, rather than a direct average of the intersite $J$ values, as suggested also by new experimental results in $C e N i_{1-x} C u_{x}$ 11 alloys.

\section{Acknowledgments}

The numerical calculations were, in part, performed at LSC (Curso de Ciência da Computação, UFSM) and grupo de Física Estatítica-IFM, Universidade Federal de Pelotas. The authors are grateful to Prof. J. I. Espeso, Prof. J. C. Gomez-Sal and Prof. Alba Theumann. This work was partially supported by the Brazilian agencies FAPERGS (Fundação de Amparo à Pesquisa do Rio Grande do Sul) and CNPq (Conselho Nacional de Desenvolvimento Científico e Tecnológico).

\section{References}

[1] E. Miranda, V. Dobrosavljevic, G. Kotliar, Phys. Rev. Lett. 78, 290 (1997).

[2] E. Miranda, V. Dobrosavljevic, Phys. Rev. Lett. 86, 264 (2001).

[3] A. H. Castro Neto, B. A. Jones, Phys. Rev. B 62, 14975 (2000).

[4] Alba Theumann, B. Coqblin, S. G. Magalhaes, A. A. Schmidt, Phys. Rev. B 63, 54409 (2001).

[5] S. G. Magalhaes, A. A. Schmidt, Alba Theumann, B. Coqblin, Eur. Phys. J. B 30, 419 (2002).

[6] S. G. Magalhaes, A. A. Schmidt, F. M. Zimmer, Alba Theumann, B. Coqblin, Eur. Phys. J. B 34, 447 (2003).

[7] A. M. Sengupta, A. Georges, Phys. Rev. B 52, 10295 (1995).

[8] J. C. Gomez Sal, J. Garcia Soldevilla, J. A. Blanco, J. I. Espeso, J. Rodriguez Fernandez, F. Luis, F Bartolomé, J. Bartolomé, Phys. Rev. B 56, 11741 (1997).

[9] J. Garcia Soldevilla, J. C. Gomez Sal, J. A. Blanco, J. I. Espeso, J. Rodriguez Fernandez, Phys. Rev. B 61, 6821 (2000). 
[10] N. Marcano, G. M. Kalvius, D. R.Noakes, J. C. Gomez-Sal, R. Wappling, J. I. Espeso, E. Schreier, A. Kratzer, Ch. Baines, A Amato, Physica Scripta XX, 1 (2003).

[11] N. Marcano, Thesis, Un. of Santander, Spain (Feb. 2005). N. Marcano, J. I. Espeso, J. C. Gomes Sal, J. Rodriguez Fernandez, J. Herrero-Albillos, F. Bartolome, Phys. Rev. B 71, 134401 (2005).

[12] S. Majumdar, F. V. Sampathkumaran, St. Berger, M. Della Mea, H. Michor, E. Bauer, M. Brando, J. Hemberger, A. Loidl, Solid State Comm. 121 665, (2002).

[13] R. Vollmer, T. Pietrus, H. v. Lohneysen, R. Chau, M. B. Maple, Phys. Rev. B 61, 1218 (2000).

[14] V. S. Zapf, R. P. Dickey, E. J. Freeman, C. Sirvent, M. B. Maple Phys. Rev. B 65, 024437 (2002).

[15] D. J. Mattis, Phys. Lett. 56A, 421 (1977).

[16] K Binder, A. P. Young, Rev. Mod. Phys. 58, 801 (1986).

[17] E. Fradkin, B. A. Huberman, S. H. Shenker, Phys. Rev. B 18, 4789 (1978).

[18] J. P. Provost, G. Vallee, Phys. Rev. Lett. 50, 598 (1983).

[19] D. Sherrington, S Kirkpatrick, Phys. Rev. Lett. 35, 1792 (1975).

[20] J. L. van Hemmen, Phys. Rev. Lett. 49, 409 (1982).

[21] Daniel J. Amit, Hanoch Gutfreund, H. Sompolinsky, Annals of Phys. 173, 30 (1987).

[22] Daniel J. Amit, Modelling brain function. The world of attractor neural networks (Cambridge University Press, 1989).

[23] Daniel J. Amit, Hanoch Gutfreund, H. Sompolinsky, Phys. Rev. Lett. 55, 1530 (1985).

[24] Daniel J. Amit, Hanoch Gutfreund, H. Sompolinsky, Phys. Rev. A 32, 1007 (1985).

[25] P. Sollich, H. Nishmori, A. C. C. Coolen, J. Van der Sijs, J. Phys. Soc. Jpn. 69, 3200 (2000).

[26] Alba Theumann, M. Vieira Gusmao, Phys. Lett. A 105, 311 (1984).

[27] Alba Theumann, A. A. Schmidt, S. G. Magalhaes, Physica A 311, 498 (2002).

[28] Alba Theumann and B. Coqblin, Phys. Rev. B 69, 214418 (2004).

[29] J. R. Iglesias, C. Lacroix and B. Coqblin, Phys. Rev. B 56, 11820 (1997).

[30] E. Dagotto, Nanoscale Phase Separation and Colossal Magnetoresistence (Springer-Verlag, Berlin, 2002). 